\title{
Choosing Wisely Canada ${ }^{\circledR}$ : Five tests, procedures and treatments to question in Emergency Medicine
}

Amy H. Y. Cheng, MD, MBA*; Sam Campbell, MB, BCh ; Lucas B. Chartier, MDCM, MPH ; Tom Goddard, $\mathrm{MD}^{\dagger}$; Kirk Magee, MD, $\mathrm{MSc}^{\dagger}$; Jill McEwen, $\mathrm{MD}^{\S}$; Atul K. Kapur, MD, MSc ${ }^{\text {; }}$ Brian R. Holroyd, MD, MBA ${ }^{\| t+}$; Suneel Upadhye, MD, MSc ${ }^{\ddagger \ddagger}$; Stephanie Couperthwaite, BA"; Brian $\mathrm{H}$. Rowe, MD, MSc ${ }^{\| * *^{\dagger \dagger}}$

\section{ABSTRACT}

Objectives: Choosing Wisely Canada (CWC) is an initiative to encourage patient-physician discussions about the appropriate, evidence based use of medical tests, procedures and treatments. We present the Canadian Association of Emergency Physicians' (CAEP) top five list of recommendations, and the process undertaken to generate them.

Methods: The CAEP Expert Working Group (EWG) generated a candidate list of 52 tests, procedures, and treatments in emergency medicine whose value to care was questioned. This list was distributed to CAEP committee chairs, revised, and then divided and randomly allocated to 107 Canadian emergency physicians (EWG nominated) who voted on each item based on: action-ability, effectiveness, safety, economic burden, and frequency of use. The EWG discussed the items with the highest votes, and generated the recommendations by consensus.

Results: The top five CAEP CWC recommendations are: 1) Don't order CT head scans in adults and children who have suffered minor head injuries (unless positive for a validated head injury clinical decision rule); 2) Don't prescribe antibiotics in adults with bronchitis/asthma and children with bronchiolitis; 3) Don't order lumbosacral spinal imaging in patients with non-traumatic low back pain who have no red flags/pathologic indicators; 4) Don't order neck radiographs in patients who have a negative examination using the Canadian C-spine rules; and 5) Don't prescribe antibiotics after incision and drainage of uncomplicated skin abscesses unless extensive cellulitis exists.

Conclusions: The CWC recommendations for emergency medicine were selected using a mixed methods approach. This top 5 list was released at the CAEP Conference in June 2015 and should form the basis for future implementation efforts.

\section{INTRODUCTION}

\section{Background}

Choosing Wisely ${ }^{\circledR}$ is a resource stewardship initiative started by the American Board of Internal Medicine Foundation in 2012. Choosing Wisely ${ }^{\circledR}$ encourages discussions between physicians and patients regarding the necessity of medical tests, procedures, and treatments, whose use may lead to unnecessary harm to patients and additional costs to the healthcare system. ${ }^{1}$ Since its inception, more than 80 specialist societies in the United States have developed lists of five commonly ordered tests or procedures used in their practice, whose necessity should be questioned. The American College of Emergency Physicians (ACEP) was a late recruitment in the Choosing Wisely ${ }^{\circledR}$ program; however, since its initial participation, ACEP has released two lists containing a total of 10 recommendations. $^{2}$

Choosing Wisely Canada ${ }^{\circledR}$ (CWC) was launched in 2014 in partnership with the Canadian Medical Association. ${ }^{3}$ Since April 2014, more than 35 medical specialty societies have developed more than 40 lists of top five tests or procedures to question in their practice. In the spring of 2014, the Canadian Association of Emergency Physicians (CAEP) was invited to formulate a top five list for CWC. CAEP elected to seek volunteers

From the *Division of Emergency Medicine, Department of Medicine, University of Toronto, Toronto, ON; †Department of Emergency Medicine, Dalhousie University, Halifax, NS; ¥Department of Emergency Medicine, University Health Network, Toronto, ON; §Department of Emergency Medicine, University of British Columbia, Vancouver, BC; ๆDepartment of Emergency Medicine, University of Ottawa, Ottawa, ON; IDepartment of Emergency Medicine and **School of Public Health, University of Alberta, Edmonton, AB; ††Alberta Health Services, Edmonton, AB; and the $\ddagger \ddagger D i v i s i o n$ of Emergency Medicine, Department of Medicine, McMaster University, Hamilton, ON.

Correspondence to: Dr. Amy H. Y. Cheng, 30 Bond Street, 1-008 Shuter, Toronto, ON M5B1W8; Email: ChengAm@smh.ca 
for its CWC Expert Working Group (EWG) to develop its top five list through a rigorous scientific process.

Emergency physicians make critical, time-pressured decisions about the diagnosis and treatment of patients who are often acutely ill or injured. The emergency department (ED) is the point of entry into the healthcare system for many patients, and ED performance (i.e., ED wait times and length of stay times) is often at the center of government and public attention. CWC is particularly relevant for emergency medicine, because emergency physicians play critical roles in utilizing and allocating finite healthcare resources. ${ }^{4}$

There has been a wealth of recent literature that questions the utility and potential harm of investigations and treatments that are commonly performed in EDs, such as medical imaging and antibiotic overuse. Studies on ED medical imaging utilization for example, revealed that Computed Tomography (CT) utilization has doubled over the past two decades in North America; however, it remains uncertain as to whether this increase in medical imaging utilization improved patient outcomes and quality of care. ${ }^{5-7}$ In fact, physicians are increasingly concerned about the potentially unnecessary exposure to radiation for all patients, especially those receiving a CT scan, and the additional cost incurred by unwarranted medical imaging studies. ${ }^{7-9}$ As a result, professional bodies in emergency medicine (ACEP) and radiology (American College of Radiology, Canadian Association of Radiologists) have begun to advocate for reducing potentially costly and harmful medical imaging tests in emergency departments, such avoiding CT head scans for patients with minor head injury and simple headache, imaging of the lumbar spine for patients with low back pain, and radiography of the ankle without clinical signs of fracture. ${ }^{8,10}$

A recent study using a large national database in the United States showed that in 2011, a staggering 265.5 million courses of antibiotics were prescribed (equivalent to 842 prescriptions per 1000 people). ${ }^{11}$ Appropriate prescription of antibiotics is necessary to curb antibiotics resistance in communities, and to reduce unnecessary patient harms such as allergies, rash, and antibiotic-induced diarrhea.

CAEP strongly supports the CWC initiative, and has joined its peers across North America in decrying excesses in medicine. In this article, the development of the list of top five tests, procedures and treatments whose use Canadian emergency physicians should question is described. For these five, there is sufficient evidence to demonstrate that the potential harms and costs outweigh any potential benefits provided by the tests, procedures or treatments.

\section{METHODS}

\section{Study design, study population and setting}

A nominal technique and modified-Delphi process were employed to generate the list of top five CWC items for Emergency Medicine. ${ }^{12,13}$ We sought the participation of Canadian emergency physicians (CAEP and nonCAEP members) from diverse backgrounds with respect to demographics, training, location of practice, types of practice, and duration of practice. Emergency physicians who do not currently practice in Canada were excluded.

\section{Phase 1: Initial list generation}

The CAEP Choosing Wisely Expert Working Group (EWG) was formed in March 2014. The EWG was tasked with identifying an initial list of common emergency department tests, procedures and treatments that were thought to be of limited value in emergency practice and whose use could be easily reduced without compromising care. A priori, the EWG decided that the CWC recommendations would be selected based on five guiding principles of (in order of descending importance): action-ability by emergency physicians, effectiveness, safety, economic burden and frequency of use. Figure 1 lists the five guiding principles and their definitions.

The EWG was comprised of ten volunteer Canadian emergency physicians from academic and community EDs, whose practices include adult only or a mixture of adults and paediatric patients. Each EWG member invited three additional experts (adult and paediatric emergency physicians) to provide further input into expanding and refining the initial list. These 30 additional experts were selected because they were thought to be able to provide insight into the initial list generation based on their type of practice (adults, mixed adult/paediatrics), location of practice (urban teaching, urban community, rural), type of training (FRCPC, CCFP-EM, etc.) and years in practice. The experts were not required to work a minimum FTE equivalent to participate. 


\begin{tabular}{|l|l|l|}
\hline 1 & Action-ability & $\begin{array}{l}\text { Recommendations should be investigations, treatments, and procedures } \\
\text { that are within the control of the emergency physician }\end{array}$ \\
\hline 2 & Effectiveness & Recommendations should be supported by evidence \\
\hline 3 & Safety & $\begin{array}{l}\text { Recommendations should aim to reduce potential harm to patients (i.e., } \\
\text { pain, exposure to radiation, time in the ED, etc.) }\end{array}$ \\
\hline 4 & Economic burden & $\begin{array}{l}\text { Recommendations should aim to reduce costs and/or economic burden on } \\
\text { the health care system }\end{array}$ \\
\hline 5 & Frequency of use & $\begin{array}{l}\text { Recommendations should be based on investigations or procedures that } \\
\text { are commonly used in the ED }\end{array}$ \\
\hline
\end{tabular}

Figure 1. Five guiding principles for CWC list generation for emergency medicine.

\section{Phase 2: Topic generation from CAEP section and committee chairs}

Further suggestions for potential CWC items were solicited via email survey to the 13 chairs of the CAEP sections, standing committees and practice committees related to practice, evidence-based medicine, or quality of care (Appendix A). Using a modified Dillman technique, the chairs were asked whether each of the candidate items in the list generated from phase 1 should be included in the final CWC list. ${ }^{14}$ The chairs were also invited to provide suggestions for any other topics that they feel should be included in the CWC list that has not been stated. The survey responses were analysed, the CWC candidate variables that received less than $50 \%$ approval from this group were removed from the initial list, and any new potential variables were added. The CAEP Chair survey is included as Supplement 1.

\section{Phase 3: List refinement by Topic Refinement Panel (TRP)}

In phase 3, a bootstrap technique was applied, and each member on the EWG identified approximately ten additional emergency physician colleagues (who were not part of phase 2) from across Canada to provide input on refining the list generated from phase 2 . The EWG attempted to engage emergency physicians from provinces not represented on the EWG (i.e., NFLD, $\mathrm{SK}, \mathrm{MB}, \mathrm{PEI}, \mathrm{NB}$ ) through personal contacts.

To ensure that the demographics of the TRP members were representative of those of emergency physicians from across Canada, demographic information from each TRP member was collected including: age, sex, training (CCFP-EM, FRCPC, other), years in practice, location of main practice (academic, urban, rural), type of practice (adults, mixed adult/paediatrics), and location (province).
The candidate variables from phase 2 were randomly divided into two sets and sent to equal groups of the TRP. Each TRP member ranked each of the candidate variables using a seven-item Likert scale based on the five guiding principles of: action-ability, effectiveness, safety, economic burden and frequency of use. An example of the TRP survey question is included as Supplement 2.

\section{Phase 4: List reduction by Expert Working Group} The median overall scores were calculated with interquartile range $(\mathrm{IQR})$ and similar median $(\mathrm{IQR})$ score calculations were obtained for each of the five questions for each item from the TRP surveys in phase 3. The top 15 candidate items were identified using the following criteria:

1) Included items with the 15 highest total median scores;

2) From the list of 15, items with median scores of five or less in any of the five questions based on the guiding principles were eliminated;

3) From the remaining items, the top eight were selected based on highest scores (and the most narrow IQR) in the five questions based on the guiding principles in the following order (from highest to lowest order of importance): action-ability, effectiveness, safety, economic burden, and frequency of use.

\section{Phase 5: Final CWC recommendations generation} The survey results and the top eight CWC items generated from phase 4 were presented at a teleconference to the EWG. Consensus was obtained following a thorough discussion, if $100 \%$ agreement was not reached for a particular item, the working group generated more discussion until $100 \%$ agreement was 
achieved and the top five CWC for emergency medicine were finalized.

Explanatory statements were written for each of the recommendation by the EWG. Where applicable, the statements were sent to experts in the field to ensure the statements' appropriateness and accuracy.

Figure 2 depicts the steps used for selecting the top five emergency medicine recommendations for Choosing Wisely Canada.

\section{Ethics}

The Health Research Ethics Board (HREB) at the University of Alberta approved the survey protocol. Consent was implied with survey completion. While physician information was requested, no identifiers were used in the analysis and the data are reported in aggregate.

\section{RESULTS}

\section{Initial list generation}

An initial list of 52 candidate variables were generated using the opinions of the CAEP EWG and the opinions of 30 other Canadian emergency physicians identified via a bootstrap technique by the EWG.

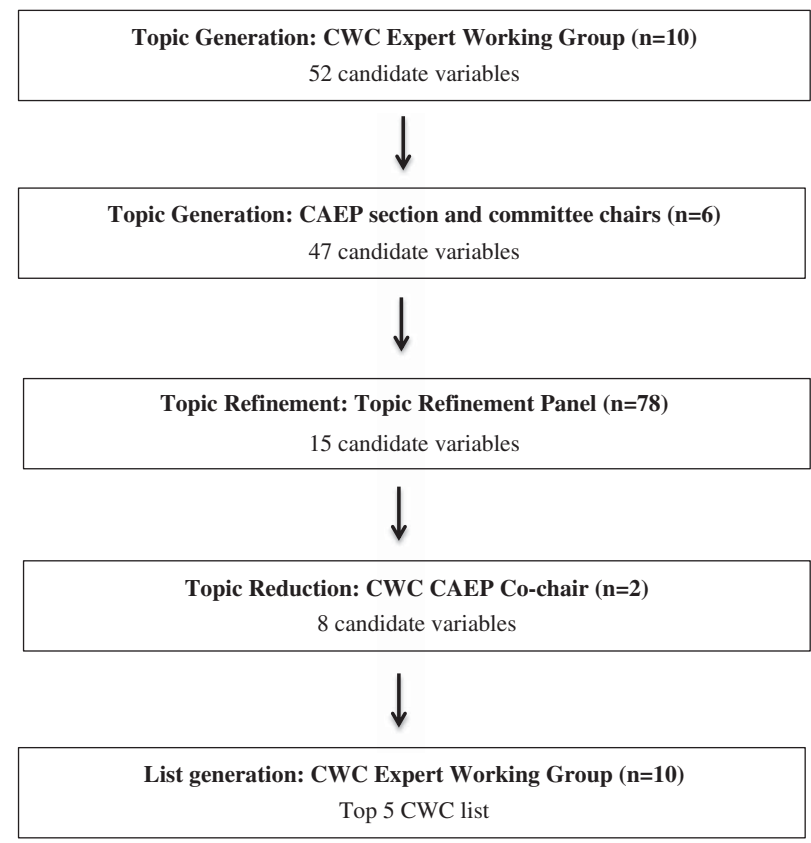

Figure 2. Method for selecting the top five Choosing Wisely Canada recommendations for emergency medicine

\section{Topic generation from CAEP section and committee chairs}

The survey with the initial list of 52 candidate variables was sent to 15 CAEP committee chairs. With a total of three reminder e-mails, six (40\%) Chairs responded. One new CWC candidate variable was suggested from this group, and five candidate variables were removed because they received less than $50 \%$ support from this group. A final list of 47 candidate variables were generated from this phase of the study.

\section{List refinement by Topic Refinement Panel}

The list of 47 candidate variables were randomly divided and sent to two groups of CAEP members comprising the TRP. Of the 107 nominated TRP members, $78(73 \%)$ completed the full survey following three email reminders. Table 1 presents the demographical

\begin{tabular}{lc} 
Table 1. Demographic information of the Topic Refinement \\
Panel \\
\hline Factor & Results \\
\hline Median age (years, [IQR]) & $46(40,54)$ \\
Female sex (n [\%]) & $24(31 \%)$ \\
Training & \\
CCFP(EM) & $37(47 \%)$ \\
FRCPC & $29(37 \%)$ \\
ABEM & $1(1 \%)$ \\
Other & $10(13 \%)$ \\
Years of practice (median [IOR]) & $15(8,27)$ \\
Type of practice & \\
Mixed Adults/Pediatrics & $44(56 \%)$ \\
Pediatrics Only & $0(0 \%)$ \\
Adults Only & $30(38 \%)$ \\
No Response & $4(5 \%)$ \\
Location of practice & \\
Urban (Academic) & $64(82 \%)$ \\
Rural & $7(9 \%)$ \\
Urban (Non-teaching) & $3(4 \%)$ \\
No Response & $4(5 \%)$ \\
Province of Practice & \\
Ontario & $21(27 \%)$ \\
Quebec & $14(18 \%)$ \\
Saskatchewan & $8(10 \%)$ \\
Manitoba & $8(10 \%)$ \\
Alberta & $7(9 \%)$ \\
British Columbia & $7(9 \%)$ \\
Nova Scotia & $5(6 \%)$ \\
New Brunswick & $2(3 \%)$ \\
Newfoundland & $2(3 \%)$ \\
Prince Edward Island & $1(1 \%)$ \\
No Response & $3(4 \%)$ \\
\end{tabular}


information of the TRP respondents. The TRP demonstrated a broad representation across Canada. Overall, the respondents were more often male (69\%), had a median age of 46 (IQR: 40,54) years, had variable certification, and a median of 15 (IQR: 8,27 ) years of practice experience.

\section{List reduction by CAEP Expert Working Group}

Overall, 15 of 47 candidate variables received a median composite score of 30 out of a possible 35 . The top eight CWC candidate variables received a median score of 6 out of 7 (IQR: 6, 7) on action-ability. Following this ranking, five received a median score of 6 (IQR: 6,7$)$ in the evidence domain. Finally, two received a median score of 6 (IQR: 6,7 ) in the safety domain, and the highest ranked CWC candidate variable received a median score of 6 (IQR: 6, 7) in the domain of economic burden (see Table 2).

\section{Final CWC recommendations generation}

The EWG reviewed the survey results and the top eight CWC items were generated. Four of the items were combined into two based on similarities: "Avoid antibiotics in children with acute bronchiolitis" was combined with "avoid antibiotics in patients with acute bronchitis.” In addition, “avoid ordering CT head scans

Table 2. Topic Refinement Panel survey results for the top 15 candidate variables

\begin{tabular}{|c|c|c|c|c|c|c|}
\hline & \multirow[b]{2}{*}{ Variable } & \multicolumn{5}{|c|}{ Median scores (IQR) for each of the five guiding principles } \\
\hline & & Action-ability & Evidence & Safety & Economic Burden & Frequency \\
\hline 1 & $\begin{array}{l}\text { Avoid ordering CT head scans in patients who have } \\
\text { suffered a minor head injury (unless positive for the CCT } \\
\text { head rule). }\end{array}$ & $6(6,7)$ & $6(6,7)$ & $6(6,7)$ & $6(6,7)$ & $6(6,6.5)$ \\
\hline 2 & Avoid antibiotics in children with acute bronchiolitis. & $6(6,7)$ & $6(6,7)$ & $6(6,7)$ & $6(5,7)$ & $6(5,7)$ \\
\hline 3 & $\begin{array}{l}\text { Avoid ordering L/S spinal views in patients with non-traumatic low } \\
\text { back pain who have no red flags. }\end{array}$ & $6(6,7)$ & $6(6,7)$ & $6(5,7)$ & $6(5,6.25)$ & $6(5,6)$ \\
\hline 4 & $\begin{array}{l}\text { Avoid ordering neck x-rays in patients who have a negative } \\
\text { examination using the Canadian C-spine rules. }\end{array}$ & $6(6,7)$ & $6(6,7)$ & $6(5,6.75)$ & $6(5,6.75)$ & $6(5,6)$ \\
\hline 5 & Avoid antibiotics in patients with acute bronchitis. & $6(6,7)$ & $6(6,7)$ & $6(5.5,6)$ & $6(5.5,7)$ & $6(5.5,7)$ \\
\hline 6 & $\begin{array}{l}\text { Avoid ordering CT head scans in pediatric patients who have } \\
\text { suffered low risk head injuries (unless positive for the } \\
\text { Catch Rule). }\end{array}$ & $6(6,7)$ & $6(5.5,7)$ & $6(6,7)$ & $6(5.25,7)$ & $6(4.25,6)$ \\
\hline 7 & Avoid ordering CT head scans among ED syncope patients. & $6(6,7)$ & $6(5,6.75)$ & $6(5,7)$ & $6(5,7)$ & $6(4.25,6)$ \\
\hline 8 & $\begin{array}{l}\text { Avoid antibiotics in incision and drainage of abscess unless } \\
\text { extensive cellulitis exists. }\end{array}$ & $6(6,7)$ & $6(5.75,7)$ & $6(5,7)$ & $6(5,6.25)$ & $6(5,7)$ \\
\hline 9 & $\begin{array}{l}\text { Avoid imaging until decision rule (e.g., Wells) +/- D-dimer testing } \\
\text { completed. }\end{array}$ & $6(5,6)$ & $6(6,7)$ & $6(6,7)$ & $6(6,7)$ & $6(5,7)$ \\
\hline 10 & $\begin{array}{l}\text { Avoid antibiotics or use the wait and see treatment strategy in } \\
\text { patients with uncomplicated sore throat }\end{array}$ & $6(5,7)$ & $6(6,7)$ & $6(6,7)$ & $6(5,7)$ & $6(5,7)$ \\
\hline 11 & $\begin{array}{l}\text { Avoid ordering ankle and/or foot radiographs in patients who have a } \\
\text { negative examination using the Ottawa ankle/foot rules. }\end{array}$ & $6(5,6)$ & $6(6,7)$ & $6(5,6)$ & $6(5,6.25)$ & $6(5.75,7)$ \\
\hline 12 & $\begin{array}{l}\text { Avoid antibiotics or use the wait and see treatment strategy in } \\
\text { patients with uncomplicated acute otitis media. }\end{array}$ & $6(5,7)$ & $6(6,7)$ & $6(5,6)$ & $6(5,7)$ & $6(5,7)$ \\
\hline 13 & $\begin{array}{l}\text { Avoid admission of patients with community acquired pneumonia } \\
\text { unless hypoxic, pneumonia severity scores }>90 \text { or social reason } \\
\text { for admission. }\end{array}$ & $6(5,6)$ & $6(5,6)$ & $6(5,6)$ & $6(6,7)$ & $6(4,6)$ \\
\hline 14 & Avoid Foley catheter insertion in all patients unless indicated. & $6(5,6.5)$ & $6(5,6)$ & $6(5,7)$ & $6(5,6.5)$ & $6(5,7)$ \\
\hline 15 & $\begin{array}{l}\text { Limit ordering chest radiographs in patients with asthma unless } \\
\text { indicated (i.e., suspected pneumothorax, pneumonia, failure } \\
\text { to respond). }\end{array}$ & $6(6,6.5)$ & $6(5,6)$ & $6(5,6)$ & $6(5,6)$ & $6(5,6)$ \\
\hline
\end{tabular}




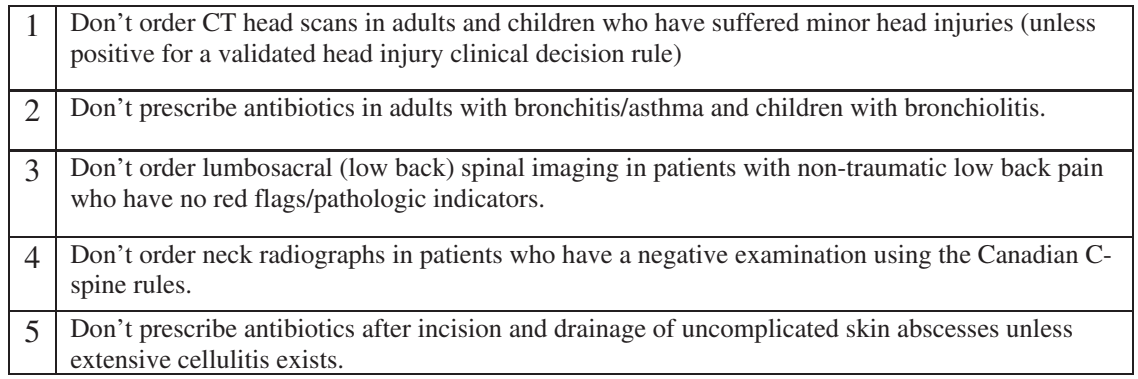

Figure 3. Choosing Wisely Canada top-5 list for emergency medicine.

in patients who have suffered a minor head injury (unless positive for the Canadian CT head rule)" was combined with "avoid ordering CT head scans in paediatric patients who have suffered low risk head injuries (unless positive for the Catch Rule)." The results of the top eight items were reviewed and the final CAEP CWC top five list was generated by consensus (see Figure 3). The products of selection were processed and refined using standard language by the CWC national committee into French and English version for posting and distribution (Appendices B and C). The CAEP CWC top five list was released after the Plenary Session presented by Dr. Wendy Levinson at the CAEP annual scientific conference in Edmonton $\mathrm{AB}$ on June 2, 2015.

\section{DISCUSSION}

The Choosing Wisely Canada campaign encourages discussions between physicians and patients regarding the necessity of medical tests, procedures, and treatments, whose use may lead to unnecessary patient harm and incur additional costs to the healthcare system. Emergency physicians make critical, timepressured decisions about the diagnosis and treatment of patients who are often acutely ill or injured. The ED is often the point of entry for patients into the Canadian healthcare system, and ED performance and throughput are often at the center of government and public attention. Thus, emergency physicians play a critical role in utilizing and allocating healthcare resources. $^{4}$

CAEP's CWC Expert Working Group used a mixed-method, consensus building approach to generate the top five CWC recommendations for emergency medicine.
Recommendation 1: Don't order CT head scans in adults and children who have suffered minor head injuries (unless positive for a validated head injury clinical decision rule)

Head injuries in children and adults are common presentations to the emergency department. Minor head injury is characterized by: Glasgow Coma Scale (GCS) 13-15, associated with either witnessed loss of consciousness, definite amnesia, or witnessed disorientation. Most adults and children with minor head injuries do not suffer from serious brain injuries that require hospitalization or surgery. CT head scans performed on patients without signs of significant injuries can expose patients to unnecessary ionizing radiation that has the potential to increase patients' lifetime risk of cancer. They also increase length of stay and misdiagnosis. There is strong evidence that physicians should not order CT head scans for patients with minor head injury unless validated clinical decision rules suggest otherwise (i.e., Canadian CT head rule for adults, and CATCH or PECARN rules for children). Despite their validity, these rules are never $100 \%$ sensitive and are meant to assist and not replace, clinical judgement. ${ }^{15-17}$

\section{Recommendation 2: Don't prescribe antibiotics in adults with bronchitis/asthma and children with bronchiolitis}

Respiratory distress from bronchospasm/wheezing is a common presentation in both children (i.e., bronchiolitis/asthma) and adults (i.e., bronchitis/asthma) seen in the ED. Most patients with symptoms do not have bacterial infections that require antibiotic treatment or influence outcomes (i.e., hospitalization). Inappropriate administration of antibiotics can expose patients to unnecessary risks (i.e., allergies, rash, other side-effects) and has the potential to increase patients' risk of 
antibiotic-induced diarrhea (including infection with C. Difficile). These prescriptions also increase overall antibiotic resistance in the community, and limit the effectiveness of standard antibiotics in the treatment of legitimate bacterial infections. There is strong applied research evidence to recommend that physicians should not prescribe antibiotics in children (i.e., bronchiolitis) and adults (i.e., bronchitis and asthma) with wheezing presentations. $^{18-20}$

\section{Recommendation 3: Don't order lumbosacral (low back) spinal imaging in patients with non-traumatic low back pain who have no red flags/pathologic indicators}

Adults with non-specific lumbosacral pain, in the absence of significant trauma (i.e., car crash, acute axial load, acute hyperflexion, etc.) commonly present to the emergency department. The evaluation of patients presenting with non-traumatic low back pain should include a complete focused history and physical examination to identify "red flags" that may indicate significant pathology. These may include, but are not limited to: features of cauda equina syndrome, weight loss, history of cancer, fever, night sweats, chronic use of systemic corticosteroids, chronic use of illicit intravenous drugs, patients with first episode of low back pain over 50 years of age and especially if over 65, abnormal reflexes, loss of motor strength or loss of sensation in the legs. In the absence of red flags, physicians should not order radiological images for patients for patients presenting with non-specific low back pain. Imaging of the lower spine for symptomatic low back pain does not improve outcomes, exposes the patient to unnecessary ionizing radiation and contributes to flow delays without providing additional value..$^{21-23}$

\section{Recommendation 4: Don't order neck radiographs in patients who have a negative examination using the Canadian C-spine rules}

Neck pain resulting from trauma (such as a fall or car crash) is a common reason for people to present to the emergency department. Very few patients have a cervical spinal injury that can be detected on radiographs ("X-rays"). History, physical examination and the application of clinical decision rules (i.e., the Canadian C-spine rule) can identify alert and stable trauma patients who do not have cervical spinal injuries and therefore do not need radiography. ${ }^{24,25}$ The Canadian
C-spine rule has been validated and implemented successfully in Canadian centers, and physicians should not order imaging unless this rule suggests otherwise. ${ }^{26}$ Unnecessary radiography delays care, may cause increased pain and adverse outcomes (from prolonged spinal board immobilization), and exposes the patient to ionizing radiation without any possible benefit. This strategy will reduce the proportion of alert patients who require imaging.

\section{Recommendation 5: Don't prescribe antibiotics after incision and drainage of uncomplicated skin abscesses unless extensive cellulitis exists}

Abscesses are walled off collections of pus in soft tissue, with Staphylococcus aureus (both sensitive and resistant to methicillin) being the microbe most frequently involved. ${ }^{27}$ Most uncomplicated abscesses should undergo incision in the ED using local analgesia or procedural sedation, complete drainage and appropriate follow-up. Evidence suggests that antibiotics are not routinely required after incision and drainage of an uncomplicated abscess. ${ }^{28}$ Physicians should not prescribe antibiotics for these patients, unless the patients are immunocompromised, systemically ill, or exhibit extensive surrounding cellulitis (cellulitis that is beyond what is expected to overlay the abscess) or lymphangitis. $^{29}$

These five recommendations represent common tests, treatments, and procedures that are frequently used in Canadian EDs whose use should be questioned as there is considerable high-quality evidence to show that the potential harms outweighs any potential patient benefits from their use. Of the five recommendations, it is interesting to note that two relate to avoiding unnecessary antibiotics and three relate to avoiding unnecessary imaging. This reflects the concerns in the current literature about the potential harms of overprescribing antibiotics and over-utilizing ionizing radiation.

\section{LIMITATIONS}

There are several weaknesses that need to be discussed. First, the response rate was lower than expected from the CAEP Committee Chairs (40\%), although the 78\% response rate from the practicing physicians was higher than other CAEP surveys. Second, while we believe a representative sample of ED clinicians was targeted by 
and engaged in the process, we were unable to assess the biases of sampling due to the ethics requirements. Third, the methods used for list item generation and to reach consensus were made by the EWG co-chairs with little precedent to inform them; however, the methods used were decided on a priori and with an attempt to focus on item reduction. Fourth, the list distribution (three imaging and two antibiotics) likely over-represents some component of practice, especially in urban settings. We recognize the list under-represents pediatric conditions, and is limited in topic coverage; however, additional recommendations are planned for 2016. Finally, CWC provides a platform to start conversations about resource stewardship in the ED, and the CAEP top five list identifies those tests, procedures and treatments that emergency physicians across the country should initially focus their attention on. However, whether the CWC recommendations will eventually be translated into institutional policy and individual physicians' practice changes that can lead to improved patient outcomes remains unclear. Future work should focus on developing local strategies that can help translate the CWC recommendations into practice. A concurrent public campaign regarding appropriate use of diagnostic tests and treatments to provide the public with information analogous to Consumer Reports in the US should also be undertaken.

\section{CONCLUSION}

A robust, multi-stage consensus building technique was used to generate the CWC top five list of tests, procedures, and treatments in emergency medicine whose use should be questioned. The five recommendations are simple, practical and supported by strong evidence: 1) Don't order CT head scans in adults and children who have suffered minor head injuries (unless positive for a validated head injury clinical decision rule); 2) Don't prescribe antibiotics in adults with bronchitis/ asthma and children with bronchiolitis; 3) Don't order lumbosacral (low back) spinal imaging in patients with non-traumatic low back pain who have no red flags/ pathologic indicators; 4) Don't order neck radiographs in patients who have a negative examination using the Canadian C-spine rules; and 5) Don't prescribe antibiotics after incision and drainage of uncomplicated skin abscesses unless extensive cellulitis exists.

Future work should focus on developing local and regional strategies that can help translate the CWC recommendations into daily practice.
Acknowledgments: All authors were responsible for the conceptualization of the study. BHR and AC developed the protocol; BHR obtained HREB approval. SC, AC and BHR were responsible for data collection and data analysis. AC, SC and BHR had access to the data (including statistical reports and tables) in the study and take responsibility for the integrity of the data and the accuracy of the data analysis. AC drafted the manuscript, and all authors contributed to its revision. AC takes responsibility for the paper as a whole, on behalf of all authors. We thank the Canadian Association of Emergency Physicians (CAEP) and Alberta Innovates Health Solutions (AIHS) for providing financial support for this project. We acknowledge the Emergency Medicine Research Group $\left(\mathrm{EMeRG}^{\circledR}\right)$, and the Department of Emergency Medicine at the University of Alberta for their assistance with the ethics submission, survey administration and data analysis. We thank Dr. Lisa Hicks for her input into the initial CWC list development methodology. The CAEP EWG would like to thank Dr. Wendy Levinson and the entire Choosing Wisely Canada ${ }^{\circledR}$ team for their commitment, encouragement and support. Finally, we also thank all of the emergency physicians across Canada for their participation in the list generation process. BHR is supported by the Canadian Institutes of Health Research (CIHR) as a Tier I Canada Research Chair in Evidence-based Emergency Medicine through the Government of Canada (Ottawa, ON). SC is supported by salary funding through $\mathrm{EMeRG}^{\circledR}$ at the University of Alberta. $\mathrm{BHR}$ and BRH work for Alberta Health Services (AHS) through the Emergency Strategic Clinical Network (ESCN).

Competing interests: None declared.

\section{SUPPLEMENTARY MATERIAL}

To view supplementary material for this article, please visit https://doi.org/10.1017/cem.2017.1

\section{REFERENCES}

1. ChoosingWisely. Choosing Wisely: An initiative of the ABIM Foundation; 2012. http://www.choosingwisely.org/ about-us/ (accessed December 8, 2014).

2. ChoosingWisely. Choosing Wisely - American College of Emergency Physicians Recommendations; 2013. http://www. choosingwisely.org/societies/american-college-of-emergencyphysicians/ (accessed August 1, 2015).

3. Choosing Wisely. ChoosingWisely Canada; 2014. http://www. choosingwiselycanada.org/about/what-is-cwc/ (accessed December 8, 2014).

4. ACEP. Emergency Physician Stewardship of Finite Resources; 2010. http://www.acep.org/Clinical-Practice-Management/ Resource-Utilization-in-the-Emergency-Department-TheDuty-of-Stewardship/ (accessed November 15, 2014).

5. Broder J, Warshauer DM. Increasing utilization of computed tomography in the adult emergency department, 2000-2005. Emerg Radiol 2006;13:25-30.

6. Smith-Bindman R, Miglioretti DL, Larson EB. Rising use of diagnostic medical imaging in a large integrated health system. Health Aff 2008;27:1491-502. 
7. Rao VM. Trends in utilization rates of the various imaging modalities in emergency departments: nationwide Medicare data from 2000 to 2008. 7 Am Coll Radiol 2011;8:706-9.

8. Choosing Wisely. Canadian Association of Radiologists: Five Things Physicians and Patients Should Question. Choosing Wisely Canada; 2014. http://www.choosingwiselycanada.org/ recommendations/canadian-association-of-radiologists-2/ (accessed December 8, 2014).

9. Brenner DJ, Hall EJ. Computed tomography-an increasing source of radiation exposure. New Engl $7 \mathrm{Med}$ 2007;357:2277-84.

10. ACEP. ACEP Announces List of Tests As Part of Choosing Wisely Campaign; 2013. http://www.acep.org/ClinicalPractice-Management/ACEP-Announces-List-of-Tests-AsPart-of-Choosing-Wisely-Campaign/ (accessed December 8, 2014).

11. Hicks LA, Bartoces MG, Roberts RM, et al. US Outpatient Antibiotic Prescribing Variation According to Geography, Patient Population, and Provider Specialty in 2011. Clin Infect Dis 2015;60:1308-16.

12. Jones J, Hunter D. Qualitative research: consensus methods for medical and health services research. BMF 1995; 311:376-80.

13. Fink A, Kosecoff J, Chassin M, Brook R. Consensus Methods Characteristics and Guidelines for Use. Santa Monica: Rand Corporation; 1991.

14. Dillman DA, Smyth JD, Christian LM. Mail and Internet Surveys: The Tailored Design Method, 2nd ed. New York: John Wiley \& Sons; 2008.

15. Easter JS, Bakes K, Dhaliwal J, et al. Comparison of PECARN, CATCH, and CHALICE rules for children with minor head injury: A prospective cohort study. Ann Emerg Med 2014;64:145-52; 152 e1-5.

16. Osmond MH, Klassen TP, Wells GA, et al. CATCH: A clinical decision rule for the use of computed tomography in children with minor head injury. CMAF 2010; 182:341-8.

17. Stiell IG, Wells GA, Vandemheen K, et al. The Canadian CT Head Rule for patients with minor head injury. Lancet 2001;357:1391-6.
18. Farley R, Spurling GK, Eriksson L, Del Mar CB. Antibiotics for bronchiolitis in children under two years of age. Cochrane Database Syst Rev 2014;10:CD005189.

19. Graham V, Lasserson TJ, Rowe BH. Antibiotics for acute asthma. Cochrane Database Syst Rev 2001;3:CD002741.

20. Smith SM, Fahey T, Smucny J, Becker LA. Antibiotics for acute bronchitis. Cochrane Database Syst Rev 2014;3:CD000245.

21. Chou R, Fu R, Carrino JA, Deyo RA. Imaging strategies for low-back pain: systematic review and meta-analysis. Lancet 2009;373:463-72.

22. Chou R, Qaseem A, Snow V, et al. Diagnosis and treatment of low back pain: a joint clinical practice guideline from the American College of Physicians and the American Pain Society. Ann Int Med 2007;147:478-91.

23. Guideline for the Evidence-Informed Primary Care Management of Low Back Pain. In: Economics IoH, 2nd ed. (ed. Practice TO). Edmonton: Institute of Health Economics Toward Optimized Practice; 2011.

24. Michaleff ZA, Maher CG, Verhagen AP, et al. Accuracy of the Canadian C-spine rule and NEXUS to screen for clinically important cervical spine injury in patients following blunt trauma: A systematic review. CMA7 2012;184:E867-76.

25. Stiell IG, Clement CM, McKnight RD, et al. The Canadian C-spine rule versus the NEXUS low-risk criteria in patients with trauma. New Engl 7 Med 2003;349:2510-8.

26. Stiell IG, Clement CM, Grimshaw J, Brison RJ, Rowe BH, Schull MJ, et al. Implementation of the Canadian C-Spine Rule: prospective 12 centre cluster randomised trial. BMF 2009;339:b4146.

27. Fridkin SK, Hageman JC, Morrison M, et al. Methicillinresistant Staphylococcus aureus disease in three communities. New Engl 7 Med 2005;352:1436-44.

28. Duong M, Markwell S, Peter J, Barenkamp S. Randomized, controlled trial of antibiotics in the management of community-acquired skin abscesses in the pediatric patient. Ann Emerg Med 2010;55:401-7.

29. Stevens DL, Bisno AL, Chambers HF, et al. Practice guidelines for the diagnosis and management of skin and soft tissue infections: 2014 update by the Infectious Diseases Society of America. Clin Infect Dis 2014;59:e10-52. 